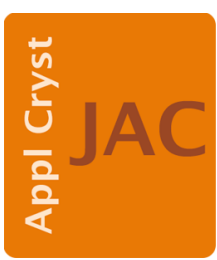

JOURNAL OF

APPLIED

CRYSTALLOGRAPHY

Volume 49 (2016)

Supporting information for article:

Evaluation of intragranular strain and average dislocation density in single grains of a polycrystal using K-map scanning

Ernesto Filippelli, Gilbert Chahine and András Borbély 


\title{
Evaluation of intragranular strain and average dislocation density in single grains of a polycrystal using K-map scanning
}

\author{
Ernesto Filippelli a, Gilbert Chahine ${ }^{\mathrm{b}}$ and András Borbély ${ }^{\mathrm{a}^{*}}$
}

${ }^{a}$ Ecole Nationale Supérieure des Mines, SMS-EMSE, CNRS:UMR 5307, LGF, 42023, Saint-Etienne Cedex 2, France

${ }^{b}$ European Synchrotron Radiation Facility, Grenoble Cedex, BP 220, France.

Correspondence e-mail: borbely@emse.fr

\section{Supplementary Material}

The peak profile presented in Fig. 3a was evaluated according to the method presented by Groma (1998) for dislocation induced peak-broadening and later by Borbély \& Groma (2001) to account for the coupled effect of dislocations and small crystal size. The equations used to describe the different order restricted moments are the following:

$$
\begin{aligned}
& M_{2}(q)=\frac{1}{\pi^{2} \varepsilon_{F}} q-\frac{L}{4 \pi^{2} K^{2} \varepsilon_{F}^{2}}+\frac{\Lambda\langle\rho\rangle \ln \left(\frac{q}{q_{0}}\right)}{2 \pi^{2}}, \\
& \frac{M_{4}(q)}{q^{2}}=\frac{1}{3 \pi^{2} \varepsilon_{F}} q+\frac{\Lambda\langle\rho\rangle}{4 \pi^{2}}+\frac{3 \Lambda^{2}\left\langle\rho^{2}\right\rangle n^{2}\left(\frac{q}{q_{1}}\right)}{4 \pi^{2} q^{2}},
\end{aligned}
$$

where $q=2\left(\sin \theta-\sin \theta_{0}\right) / \lambda$ is the reciprocal space variable measured from the centre of mass of the peak, $\lambda$ is the wavelength of the X-rays and $\left(\theta-\theta_{0}\right)$ is the angular range associated with $q$. $K$ is the Scherrer constant and $L$ is the taper parameter depending on the decrease rate of the cross sectional area of crystallites. $\varepsilon_{F}$ is the surface weighted apparent coherent domain size in a direction perpendicular to the diffracting planes. $\Lambda$ is a geometrical constant related to the strength of the dislocation contrast:

$\Lambda=\frac{\pi}{2} \mathrm{~g}^{2} b^{2} C$

where the contrast factor $C$ and was calculated using the AnizC program (Borbély et al., 2003). For the analysed grain it was considered that only the slip system (11-1)[101] with the highest Schmid factor of 0.380 is activated. Considering equal population of edge and screw dislocation $C=0.1917$.

The third order restricted moment was calculated by Groma (1998). Using the definitions in Borbély \& Groma (2001) this moment contains an extra factor of $1 /(2 \pi)^{3}$ : 
$M_{3}(q)=-\frac{3}{(2 \pi)^{3}}\left\langle P_{0}\right\rangle \ln \left(\frac{q}{q_{1}}\right)$,

where the polarization of the dislocation structure $\left\langle P_{0}\right\rangle=2 \Lambda \pi\left\langle\frac{\mathrm{g}_{l} \mathrm{~g} m}{\mathrm{~g}} \frac{\partial \varepsilon_{l}}{\partial x_{m}} \rho\right\rangle$ is related to the spatial average of the strain gradient weighted by the local dislocation density and the direction cosines of the diffraction vector, g. Repeated indices indicate summation according to the Einstein's convention.

\section{Figures}

Figure S1. The tensile stress-strain curve of the analysed Al-0.1wt.\%Mn polycrystal. The dashed line indicates the strain up to which the analysed sample was deformed

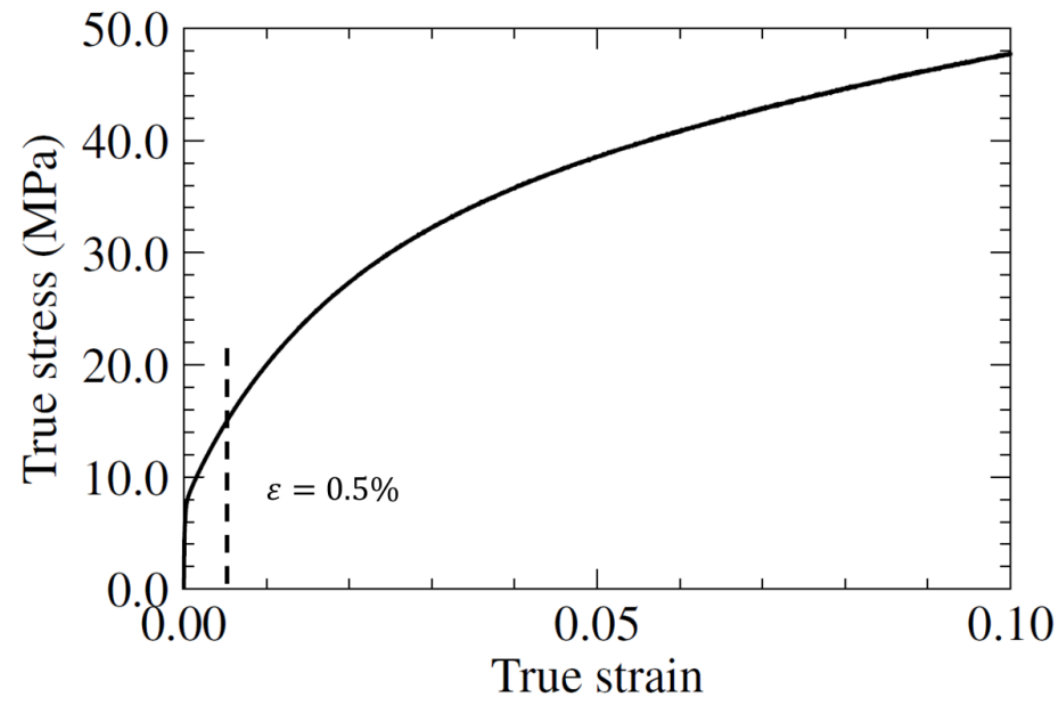

Figure S2. Band Contrast map for the analysed grain obtained with HKL Channel5 commercial EBSD software

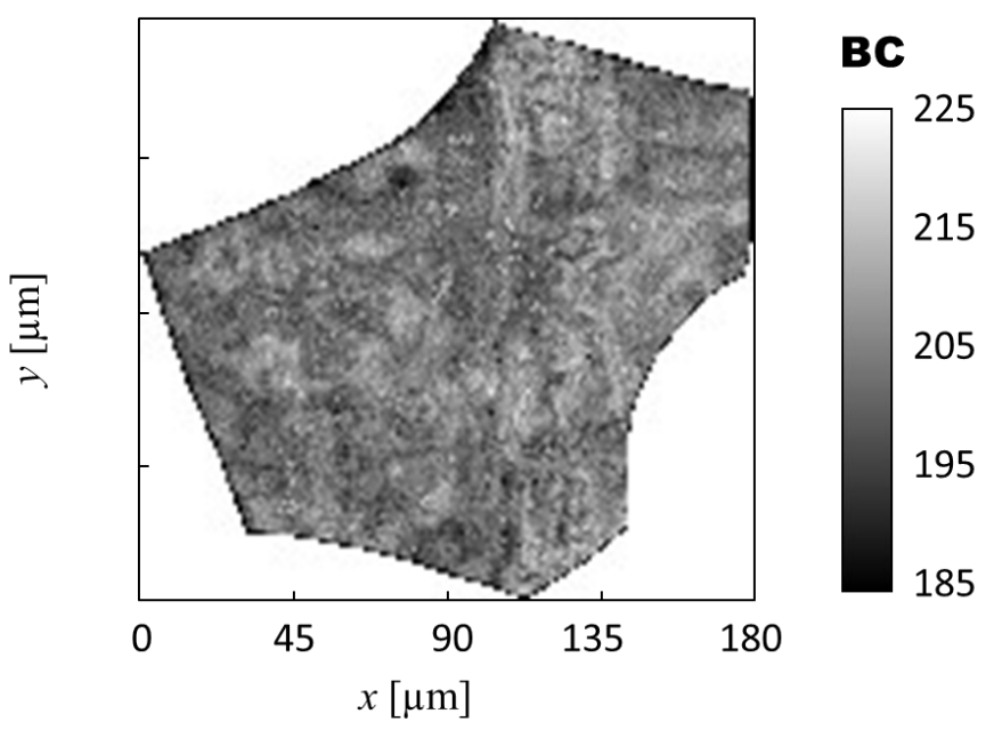


Figure S3. Distribution of the lattice tilt component $\alpha_{x}$ (the angle between the local $Q_{x}$ component and the average value of $\overline{Q_{x}}$ over all pixels) calculated from: a) K-map rocking curves and b) EBSD.

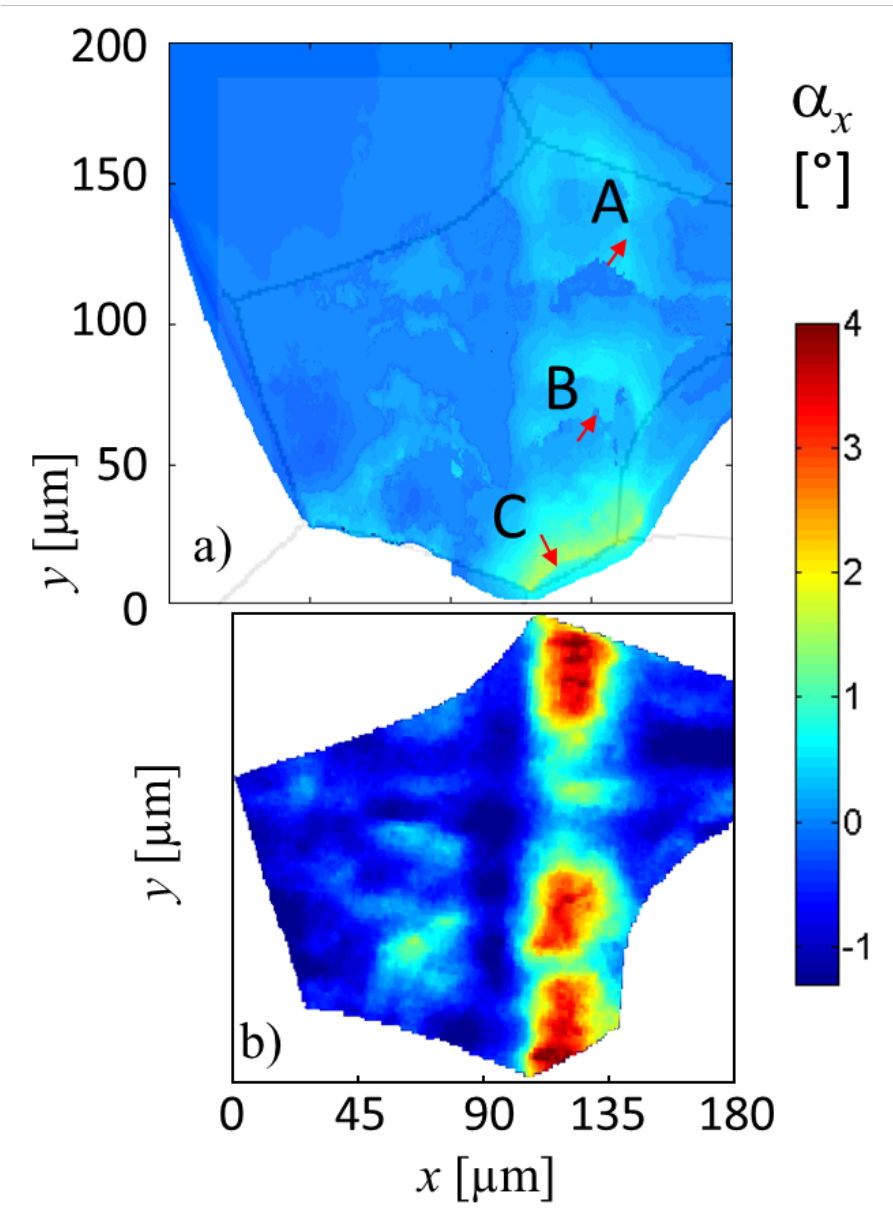

Figure S4. The fourth order restricted moment of the $Q$ distribution given in Fig. 3a. The $4^{\text {th }}$ order moment shows a much higher scatter than the variance. According to equation (E2) in the absence of small particle size, $M_{4} / q^{2}$ should tend to the constant $\frac{\Lambda\langle\rho\rangle}{4 \pi^{2}}$ at large $q$ values. A line has been fitted at the maximum of the peak, before the decrease due to cut-off.

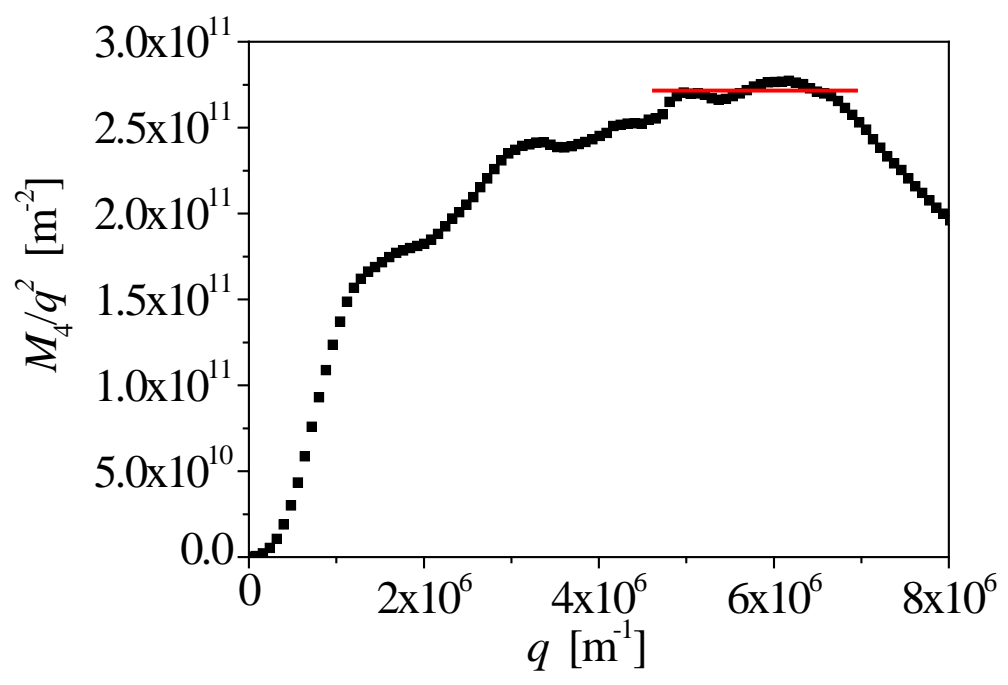


Figure S5. The $3^{\text {rd }}$ order restricted moment of the peak given in Fig. 3a as a function of $\log (q)$. The slope of the line fitted before the decrease of $M_{3}$ due to the cut-off is proportional to the polarization of the dislocation structure.

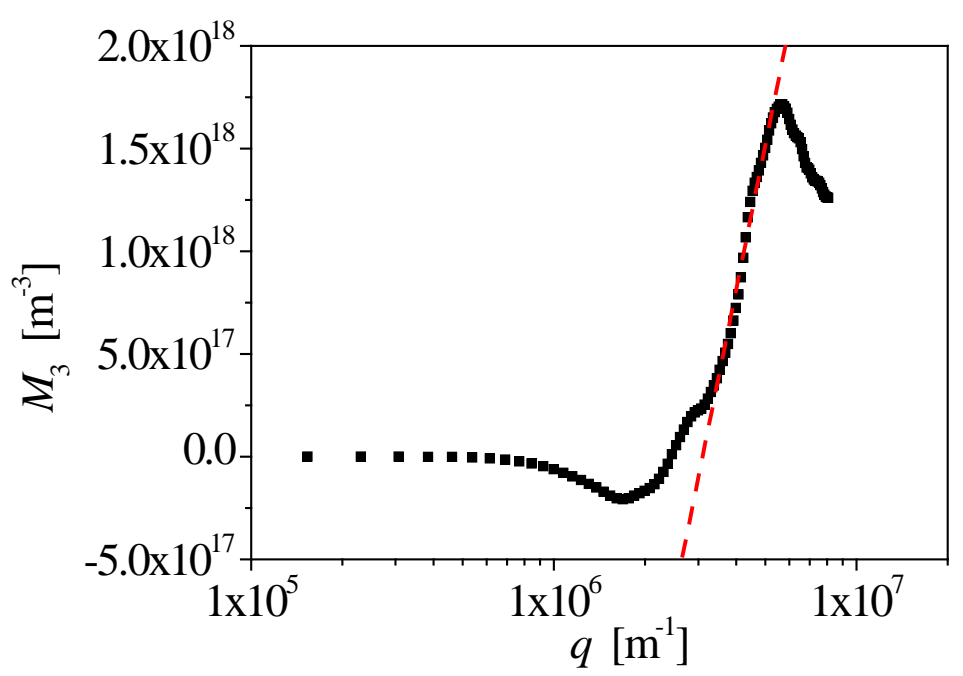

